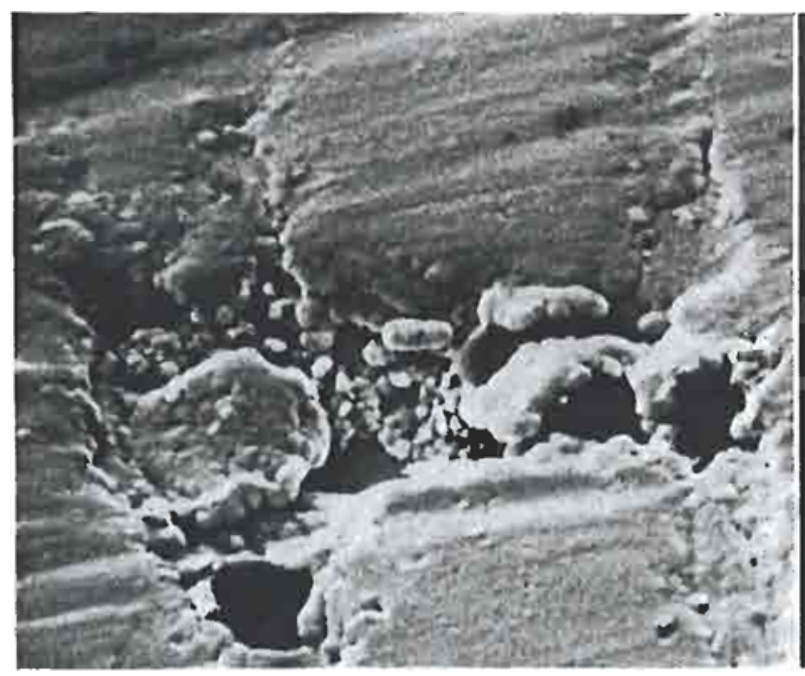

Fig. 4 Gold beginning to spread over a partiele of residual polishing compoumd in a triangular pore in 0.2 micrometre gold plate. The gold would eventually fill the pore but prohably only as a rathex spongy deposit giving unreliahle protection.

$(\times \mathbf{5 7 5 0})$

this would have grown right across the pore but there would still have been a gap beneath it. Fig. 4 illustrates another way in which gold gradually covers over a pore as the thickness of the plating builds up. The pore in Fig. 4 contains one small inclusion at the lowest point of the triangle and another in its top left-hand corner but in the right-hand corner is a rather large inclusion with gold beginning to deposit over it. The feature projecting from the left-hand side of the triangle may be a fourth inclusion almost completely covered by gold, or it may be a flap of gold growing out across the pore, similar to that shown in Fig. 3. Whichever mechanism results in eventual covering of the pore by gold, there will still be a local defect and possibly even a group of minute discontinuities in the plating where the pore was originally located.

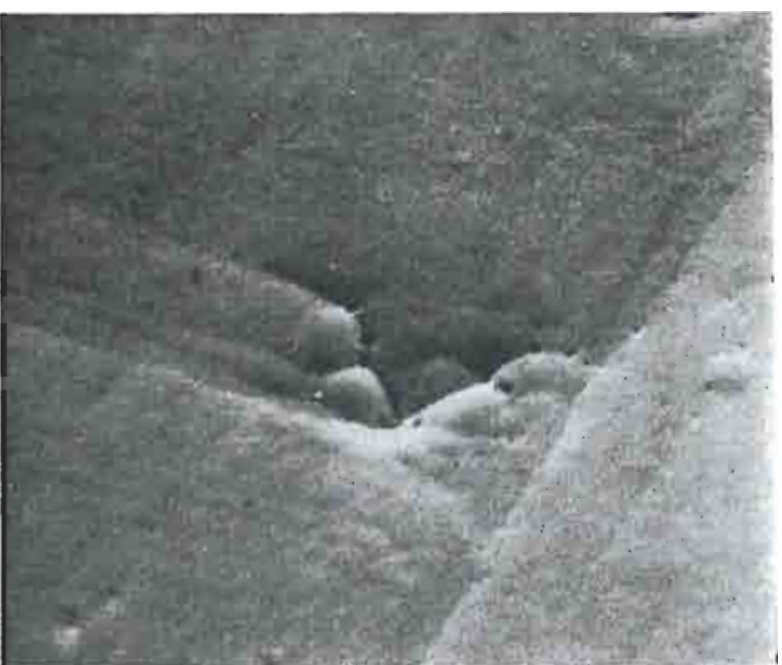

Fig. 5 A gold deposit 2.0 micrometres thick which has eovered over a pore that was present when the plating was thinner but still shows obvious weakness where the pore was situated.

$(\times \mathbf{4 5 0 0})$

Fig. 5 shows a much thicker (2 micrometre) deposit; the feature at the centre of the photograph is probably one of the triangular pores of the type shown in Figs 2, 3 and 4 now largely covered by gold but still clearly a weak point in the plating.

The SEM that was used for studying pores in gold plating incorporated a facility for making X-ray analyses on selected minute areas of specimens while in the microscope. Using this, it was shown that the inclusions associated with triangular pores were particles of polishing compound still remaining in spite of the elaborate cleaning procedure that had been adopted before plating. The difficulty of preparing surfaces free from such contamination by the normal commercial pre-plating cleaning procedures is obvious and further work is, therefore, being carried out on improved cleaning methods.

\title{
Structure of the Gold-Silicon Eutectic
}

The low melting point eutectic alloys of gold-silicon, gold-germanium and gold-tin are used as solders in electronic engineering to make electrical or thermal connection between a silicon chip and a suitable substrate, the chip being a pre-alloyed or diffused semi-conductor diode, transistor or integrated circuit. In the case of gold-silicon-the eutectic composition is at 3.25 per cent gold and the melting point $370^{\circ} \mathrm{C}-$ the eutectic is either used as a pre-form produced from strip or it is developed in situ by depositing gold on to the silicon and alloying at a temperature above the eutectic.

Studies of the microstructure of this alloy and of its modification by varying the rate of cooling from the liquid state were reported by E. Philofsky and his collaborators of Motorola Semiconductor Products to the meeting of the Electrochemical Society in October last. Cooling rates ranging from less than $\mathrm{Io} \mathrm{deg} . \mathrm{C} / \mathrm{sec}$ to more than $1000 \mathrm{deg} . \mathrm{C} / \mathrm{sec}$ were used. At the slowest rates a coarse microstructure was found comprising discrete silicon particles of various shapes in a gold matrix. At intermediate rates a cylindrical dendrite structure was observed, the dendrite spacing decreasing as cooling rate increased, while at the very high rates of cooling-obviously impracticable in actual semiconductor techniques-X-ray diffraction revealed the presence of new complex cubic structures in addition to gold and silicon. Subsequent heat treatment transformed this structure to one consisting apparently of pure gold with the silicon too fine to resolve.

These findings could possibly be helpful in the fabrication of gold-silicon eutectic alloy strip from its brittle as-cast structure by appropriate heat treatment followed by cold rolling. 